Musées, Patrimoine et Culture scientifiques et techniques

159 | 2015

mai-juin 2015

\title{
Développement durable : enjeux actuels des muséums et jardins botaniques
}

\author{
Yves-Marie Allain
}

\section{OpenEdition \\ Journals}

Édition électronique

URL : http://journals.openedition.org/ocim/1508

DOI : 10.4000/ocim. 1508

ISSN : 2108-646X

Éditeur

OCIM

Édition imprimée

Date de publication : 1 mai 2015

Pagination : 7-11

ISSN : 0994-1908

Référence électronique

Yves-Marie Allain, « Développement durable : enjeux actuels des muséums et jardins botaniques », La Lettre de l'OCIM [En ligne], 159 | 2015, mis en ligne le 01 mai 2016, consulté le 01 mai 2019. URL http://journals.openedition.org/ocim/1508; DOI : 10.4000/ocim.1508

Ce document a été généré automatiquement le 1 mai 2019.

Tous droits réservés 


\title{
Développement durable : enjeux actuels des muséums et jardins botaniques
}

\author{
Yves-Marie Allain
}

Le Jardin botanique de Dijon avec sa petite orangerie datant de la première moitié du XIX ${ }^{\mathrm{e}}$ siècle.

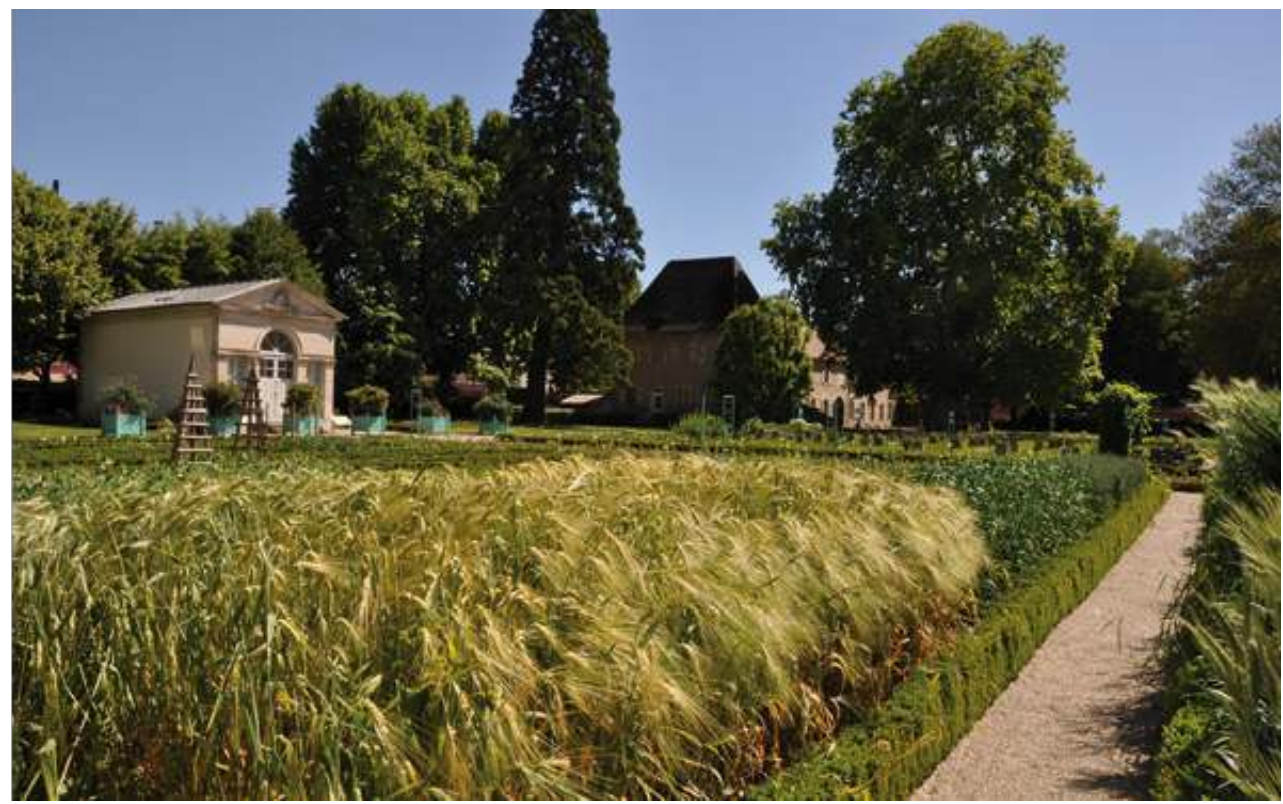

(c) Jardin des sciences de Dijon

1 Le thème " Développement durable : enjeux actuels des muséums et jardins botaniques " est si vaste qu'il ne m'est pas possible de l'envisager dans sa globalité, compte tenu de la complexité et de la diversité des sujets qui seraient à aborder. Aussi vais-je, à partir de 
mon expérience professionnelle, évoquer quelques points et poser des questions afin d'alimenter la réflexion.

\section{Un peu d'histoire}

2 Un rapide survol historique montre que les jardins botaniques et les muséums ont des origines différentes. Le jardin botanique fut créé pour cultiver des plantes afin de pouvoir les observer et les étudier vivantes, alors que le muséum reçoit des objets biologiques préparés et souvent naturalisés afin de constituer des collections inertes.

3 Les premiers jardins botaniques remontent au XVI siècle, lors de la Renaissance italienne dans des villes comme Venise, Padoue, Florence... En France, le premier jardin est celui de Montpellier en 1597, puis viennent ceux de Strasbourg en 1619, de Paris en $1626^{1}$. L'objectif de ces nouveaux foyers de science: connaitre, dénommer (nomenclature) classer et hiérarchiser (taxonomie) toutes les plantes, et ce en dehors de tout mode d'usage ou d'utilisation.

4 Les cabinets de curiosités apparaissent plus tard, au cours des XVII ${ }^{\mathrm{e}}$ et XVIII ${ }^{\mathrm{e}}$ siècles, et seront transformés au cours du XIX ${ }^{e}$ siècle en muséum. Il est à noter qu'au moment de sa création, l'Hortus publicus de Leyde (1587) possède un début de cabinet de curiosités. Dans la galerie de ce jardin botanique des Pays-Bas se trouvent pêle-mêle des animaux naturalisés dont des crocodiles, des chauves-souris, une carapace de tortue, une mâchoire d'ours, des plantes sèches, des graines, des cartes... un embryon de muséum en quelque sorte.

En France, le premier muséum sera à Paris, le «Muséum d'Histoire naturelle », nom choisi par la Convention en 1793, par modification de celui de «Jardin royal des plantes». Ce n'est qu'au cours du XIX ${ }^{e}$ siècle qu'il deviendra national pour se différencier des nombreux muséums créés en province.

6 Une autre décision de la Convention aura des répercussions à moyen terme sur la création d'un réseau français tant de jardins botaniques que de muséums. En effet, par décret du 25 février 1795, il est prévu de créer au moins une école centrale par département. Dans son article 4, la loi du 3 brumaire de l'an IV (25 octobre 1795), décrète l'obligation pour chaque école centrale de posséder une bibliothèque, un cabinet d'Histoire naturelle, un cabinet de sciences expérimentales et un jardin botanique. Beaucoup de ces cabinets et jardins disparaitront au moment de la suppression des écoles centrales remplacées, sur décision de Napoléon $1^{\text {er }}$ en 1802 , par les lycées. Mais la dynamique initiée et la création de groupes de naturalistes ne seront pas sans conséquence sur la création et le développement, au cours des $\mathrm{XIX}^{\mathrm{e}}$ et $\mathrm{XX}^{\mathrm{e}}$ siècles, des deux institutions qui nous préoccupent.

7 Si le rattachement administratif des jardins botaniques est variable (État, universités, collectivités territoriales, entreprises privées, particuliers), les muséums eux sont, pratiquement tous, municipaux².

\section{Que s'est-il passé durant ces dernières décennies ?}

En mai 2010, il m'avait été demandé d'intervenir durant un séminaire pour les élèves de dernière année de l'École nationale supérieure du paysage de Versailles. Le thème général 
était «Écologie, mentalité et savoir-faire aux jardins ». Par le jeu du calendrier, je faisais cette intervention 40 ans après avoir quitté comme élève cette même école. Une rapide analyse de la situation, certes des paysagistes, montrait que nous étions passés de la liberté de composition, de proposition, de conception y compris par des associations végétales des plus incongrues, à un monde de contraintes, de réglementations, de règles au nom de la sauvegarde des espèces indigènes, des habitats naturels... De façon simple, sous l'emprise d'une interprétation sociétale du concept de biodiversité, la liberté de création et d'emploi des végétaux s'était fortement réduite ${ }^{3}$. Cette anecdote est également révélatrice de la vitesse à laquelle ont évolué durant ces dernières décennies, les références vis-à-vis du monde vivant.

Le muséum d'Histoire naturelle : un lieu pour mesurer les enjeux de la biodiversité, découvrir la richesse et la diversité du vivant mais également sa fragilité.

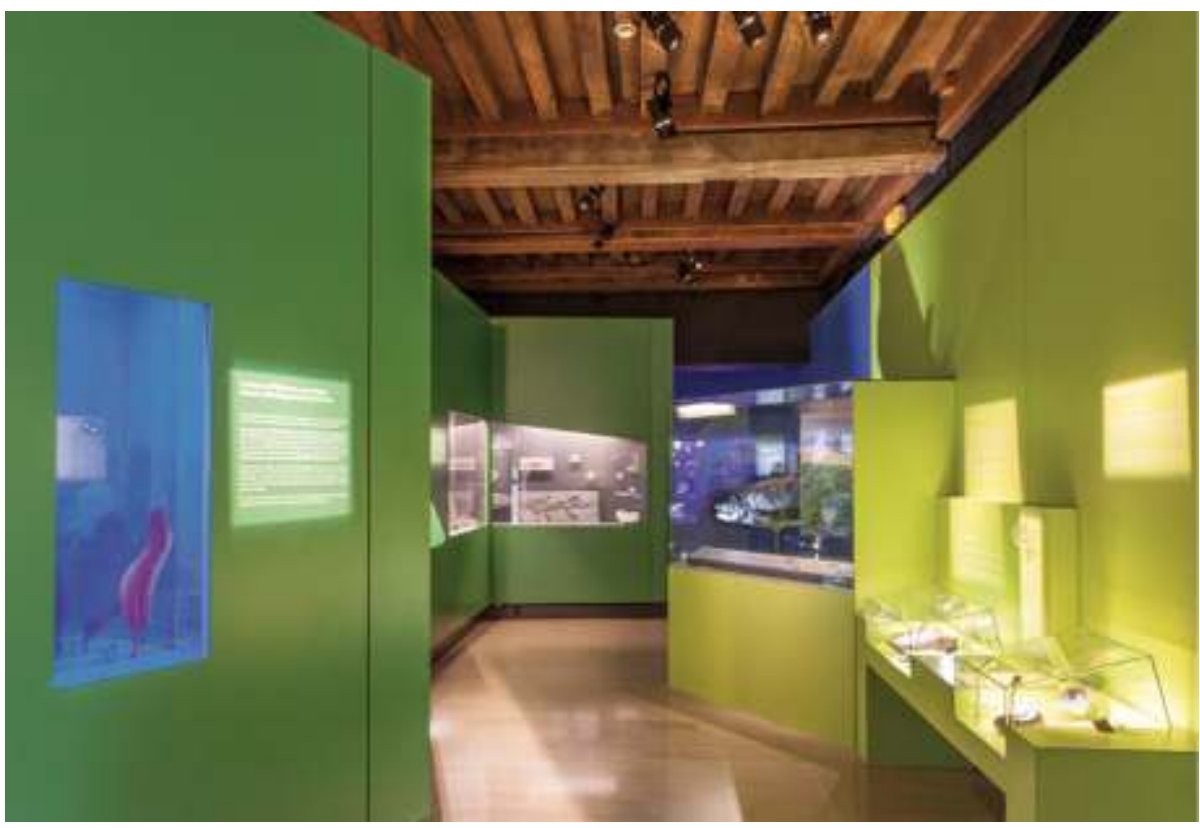

(c) Jacques Blanchard

Si en France, les premières lois de protection de la nature datent des années 1970 et s'appliquent initialement à des territoires bien définis et limités tant en nombre qu'en superficie, ce n'est qu'en 1987 que l'expression « Développement durable » apparaît pour la première fois à l'occasion de la publication du rapport Brundtland ${ }^{4}$. Ce Développement durable est défini comme "un développement qui répond aux besoins du présent sans compromettre la capacité des générations futures à répondre aux leurs». Mais cela n'a véritablement de sens que si le social en est l'objectif, l'économie un moyen et l'environnement une condition. Par ailleurs, certains auteurs considèrent que ce Développement durable est une mystification car le trépied (social, économie, environnement) n'existe pas et que ce sont en réalité des sphères concentriques ${ }^{5}$. Pour d'autres, il manque un quatrième pilier, celui culturel. Il permettrait d'introduire la notion de patrimoine, et de réhabiliter l'histoire des hommes, des échanges, des apports exogènes. Car il ne faudrait pas oublier cet autre patrimoine lié à la culture et la biologie, le patrimoine ethnologique et ethnobotanique, cette science des rapports de l'Homme avec la Nature et plus particulièrement les plantes. Parmi les éléments importants, le vocabulaire employé pour cette connaissance et dans le cadre des pratiques quotidiennes 
des divers locuteurs des langues vernaculaires, que ce soit des langues au sens strict ou des idiomes. Tous les mots utilisés non seulement désignent des objets, mais sont la concrétisation du rapport de l'homme à la nature, à la terre, voire au cosmos. Six mille langues vernaculaires sont recensées aujourd'hui, et notre siècle pourrait voir la disparition de plus de $90 \%$ d'entre elles, avec la culture qui leur est associée. Au même titre que la perte de la diversité biologique, la disparition des langues appauvrit la diversité culturelle de l'espèce humaine.

Mais les deux institutions, objet de la réflexion, ne sont-elles pas plus légitimement concernées par une autre expression apparue également dans les années 1980, celle de "diversité biologique ", c'est-à-dire "la totalité de toutes les variations de tout le vivant", selon Edward $\mathrm{O}$. Wilson (créateur du mot biodiversity) ${ }^{6}$. La première véritable définition complète mais complexe, est celle qui est formulée au cours de la XVIII assemblée générale de l'Union internationale pour la conservation de la nature (UICN), en 1988 : « $L a$ diversité biologique, ou biodiversité, est la variété et la variabilité de tous les organismes vivants. Ceci inclut la variabilité génétique à l'intérieur des espèces et de leurs populations, la variabilité des espèces et de leurs formes de vie, la diversité des complexes d'espèces associées et de leurs interactions, et celle des processus écologiques qu'ils influencent ou dont ils sont les acteurs (dite diversité écosystémique)».

11 Depuis cette date de nombreuses variantes furent données dont celle qui figure à l'article 2 de la Convention sur la diversité biologique (CDB) de 1992: "Variabilité des organismes vivants de toute origine, y compris, entre autres, les écosystèmes terrestres, marins et autres écosystèmes aquatiques et les complexes écologiques dont ils font partie; cela comprend la diversité au sein des espèces, et entre les espèces et ainsi que celle des écosystèmes ", ou celle du Conseil de l'Europe, de décembre 1996 : «La biodiversité est une notion globale qui revêt une dimension non seulement scientifique mais aussi sociale et économique. L'ensemble de la vie sur terre fait partie d'un immense système interdépendant. La biodiversité est l'expression de cette variété de vie. Omniprésente, elle s'applique aux gènes, aux espèces animales et végétales, aux écosystèmes et aux paysages de la planète ».

12 Concept scientifique avant d'être politique, les scientifiques vont investir le domaine, tenter d'expliciter leurs démarches intellectuelles de recherches et vont inclure " globalement quatre aspects dans le terme biodiversité:

13 - l'étude des mécanismes biologiques fondamentaux permettant d'expliquer la diversité des espèces et leurs spécificités et nous obligeant à davantage »décortiquer« les mécanismes de la spéciation et de l'évolution,

14 - les approches plus récentes et prometteuses en matière d'écologie fonctionnelle et de biocomplexité, incluant l'étude des flux de matière et d'énergie et les grands cycles biogéochimiques,

15 - les travaux sur la nature "utile" pour l'humanité dans ses capacités à fournir des aliments, des substances à haute valeur ajoutée pour des médicaments, produits cosmétiques, des sondes moléculaires ou encore à offrir des modèles originaux et pertinents pour la recherche fondamentale et finalisée, afin de résoudre des questions agronomiques ou biomédicales (Boeuf, 2008),

16 - la mise en place de stratégies de conservation pour préserver et maintenir un patrimoine naturel constituant un héritage naturellement attendu pour/par les générations futures.

17 La biodiversité en fait, c'est le vivant dans toute sa complexité, c'est la fraction vivante de la Nature $»^{7}$. 
Intérieur des grandes serres du Jardin botanique de Lyon.

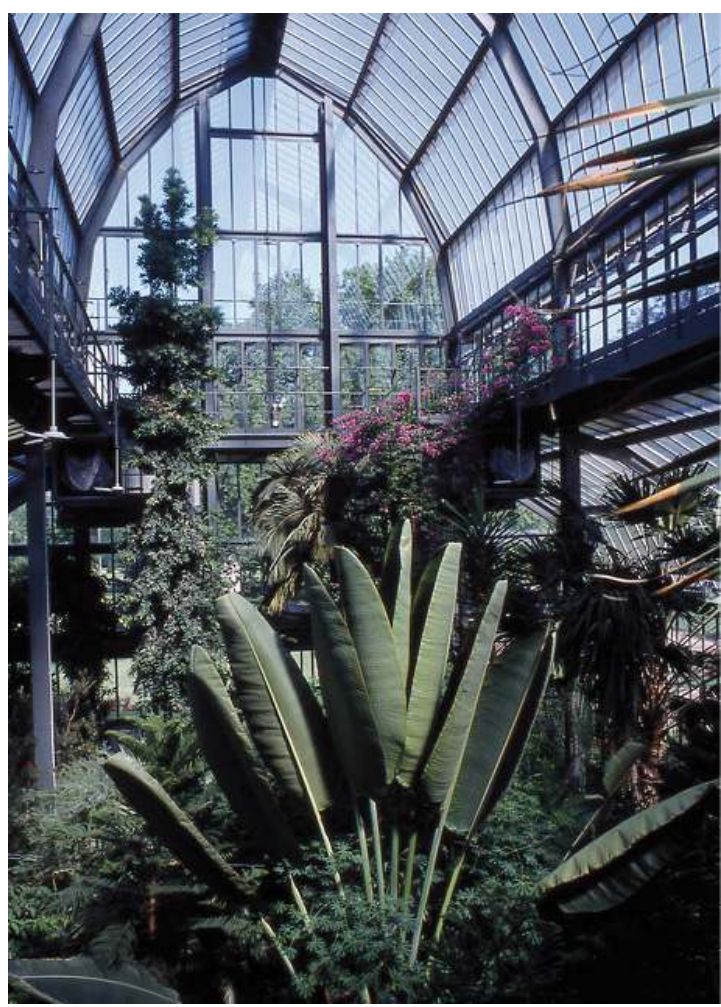

(c) Jardin botanique de Lyon

18 Mais qui sur le territoire et dans l'action quotidienne, se préoccupe de cette interdépendance, qui est porteuse de la transversalité nécessaire pour la mise en œuvre d'un tel programme, avec l'introduction des notions d'habitat et d'interaction permanente des mondes vivants et inertes? N'est-ce pas le rôle conjoint des muséums et des jardins botaniques?

19 Jusqu'à présent les jardins botaniques et les muséums étaient essentiellement orientés sur la conservation d'objets, c'est-à-dire de taxons déterminés, décrits, enregistrés et conservés sous forme de collections. Or le concept de diversité biologique apporte de grands changements. On ne doit plus uniquement conserver et protéger des objets déterminés, mais également ceux dont on n'a pas encore la preuve de leur existence, des objets non identifiés, non dénommés, et de plus il faut conserver les milieux et les habitats auxquels ils appartiennent et dans lesquels ils vivent. Il faut donc prendre en compte un patrimoine inconnu et surtout intégrer une dynamique d'évolution et de changement de ce patrimoine, sachant que vraisemblablement plus des $3 / 4$ des êtres vivants à protéger (dont ceux du sol) sont encore actuellement inconnus.

\section{Les jardins botaniques et muséums dans Le Code de l'Environnement}

Le Code de l'Environnement s'enrichit régulièrement et la nécessité d'une vision cohérente et homogène sur les critères d'évaluation de la biodiversité et de son évolution s'avère 
indispensable. La question de la place des jardins botaniques et des muséums dans ce code, partie législative et réglementaire, se trouve donc posée.

La cohérence des indicateurs lors de l'acquisition de données est devenue d'autant plus importante que les possibilités de traitements informatiques se sont développées et qu'il devient, du moins en théorie, aisé de faire des synthèses ou des regroupements thématiques.

Afin d'essayer de construire cette cohérence, la loi de 2002 relative à la démocratie de proximité précise les missions et rôles d'un certain nombre d'acteurs. Repris dans Le Code de l'Environnement, il est clairement indiqué que "l'inventaire du patrimoine naturel est institué pour l'ensemble du territoire national terrestre, fluvial et marin ». La connaissance n'est donc pas réduite aux seules zones réglementairement ou contractuellement protégées, mais doit s'étendre à tous les territoires, même ordinaires, et sur la totalité du territoire de la République, y compris donc l'Outre-Mer.

Afin d'assurer la cohérence et la synthèse des productions des divers intervenants, «ces inventaires sont conduits sous la responsabilité scientifique du Muséum national d'histoire naturelle ». Formellement, la loi n'a prévu aucune déclinaison territoriale, aucun relais, aucun appui sur les jardins botaniques ou muséums de province. Ces deux institutions n'existent pas dans Le Code de l'Environnement, ni dans sa partie législative et a fortiori encore moins dans sa partie réglementaire.

Un observateur du suivi des insectes pollinisateurs.

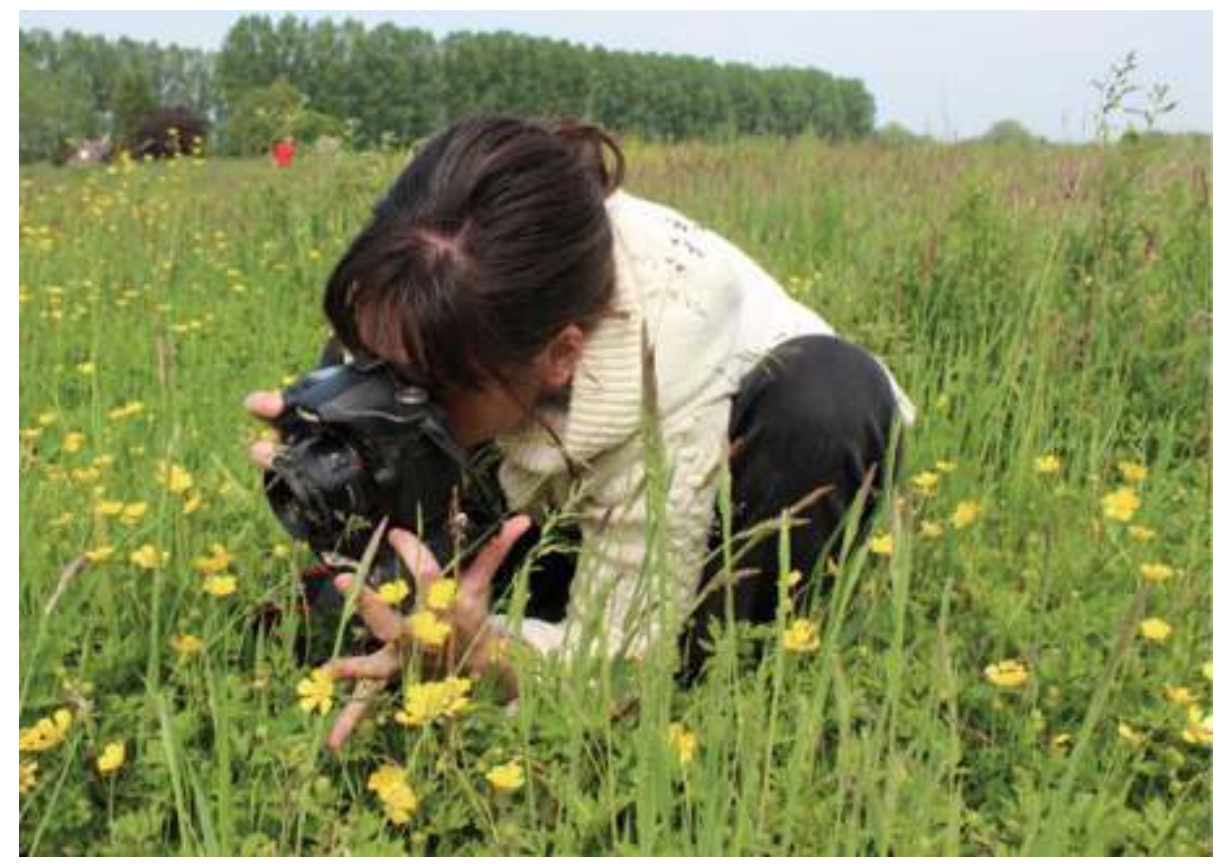

(c) MNHN/ M. Evanno

Or si les dernières lois, Grenelle I et II, (2009 et 2010) parlent de Trames vertes et bleues, pas un mot sur les jardins botaniques ni les muséums, alors que les conservatoires botaniques nationaux - il est à noter que pas un seul jardin botanique n'est actuellement agréé par l'État comme conservatoire botanique national - se trouvent renforcés dans leurs prérogatives, ainsi que quelques autres groupements de défense de la nature. 
Sous le regard des scientifiques du Biodôme de Montréal, les jeunes participent à la restauration de l'ail des bois en milieu naturel.

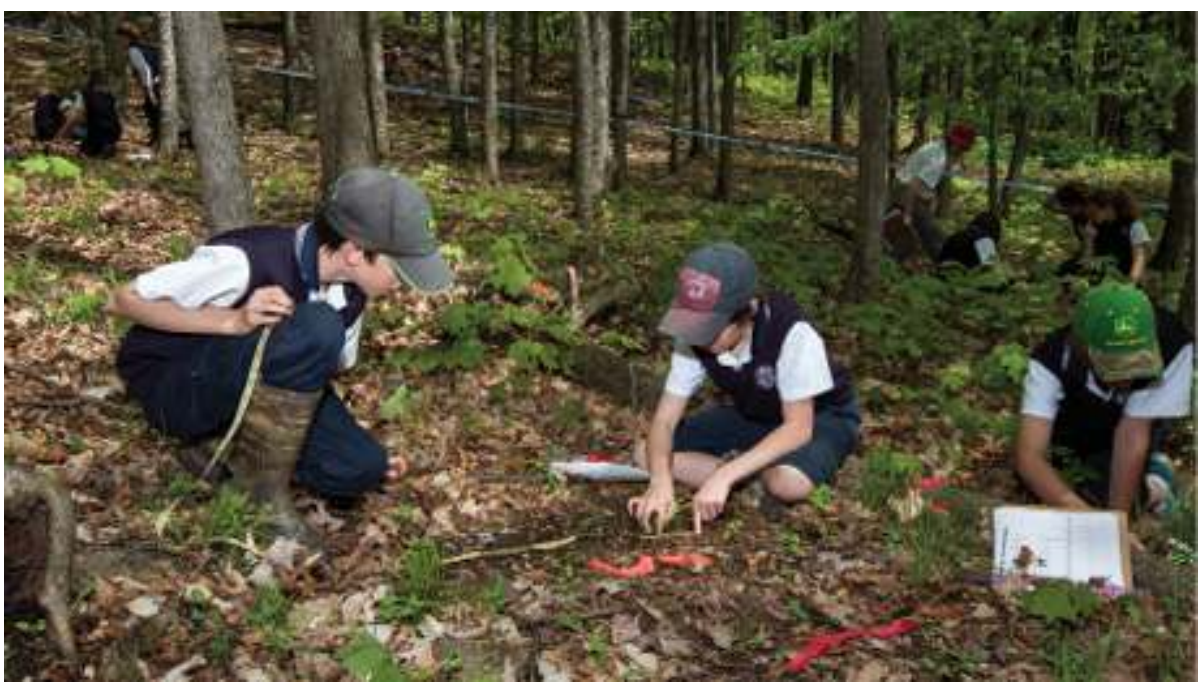

(c) Espace pour la vie

\section{Expertise}

Mais l'absence de reconnaissance des institutions, entraîne-t-elle l'absence de reconnaissance des membres de ces institutions? L'un des critères, qui serait à affiner, pourrait être la place des scientifiques des jardins botaniques et muséums dans le maillage scientifique national et local, comme par exemple le nombre de responsables siégeant dans les CSRPN (conseil scientifique régional du patrimoine naturel), dans les conseils scientifiques des réserves naturelles, dans les comités des Trames vertes et bleues, dans les déclinaisons locales de la stratégie nationale de la biodiversité, dont la seconde phase a débuté en 2011.

L'expertise ne peut exister que si des experts sont présents dans les institutions, c'est-àdire des spécialistes sur un domaine donné ou des scientifiques ayant les capacités de synthèse et de hiérarchisation des données parfois éparses et de niveaux scientifiques variables.

Mais au nom de qui est rendue cette expertise, car si dans l'enseignement supérieur, un professeur a la liberté de parole et d'écrit, du moins en théorie dans son domaine de compétence, qu'en est-il des fonctionnaires territoriaux? Ces questions ne sont pas que théoriques car la société a besoin de savoir qui parle et au nom de qui.

\section{Les mots absents}

À la lecture des documents préliminaires à ces journées de rencontres et d'études, certains mots ne figurent pas ou sont à peine cités dont ceux de science, de collections. La science ferait-elle peur au point qu'elle n'apparaisse dans le discours d'introduction que de manière sibylline tout comme le concept de diversité biologique? Il en est de même des collections dont il faudrait redéfinir la place et le rôle en tant que patrimoine scientifique, indigène et exogène, que renferment les muséums et jardins botaniques. 
Animation avec les lycées au Jardin botanique de Dijon.

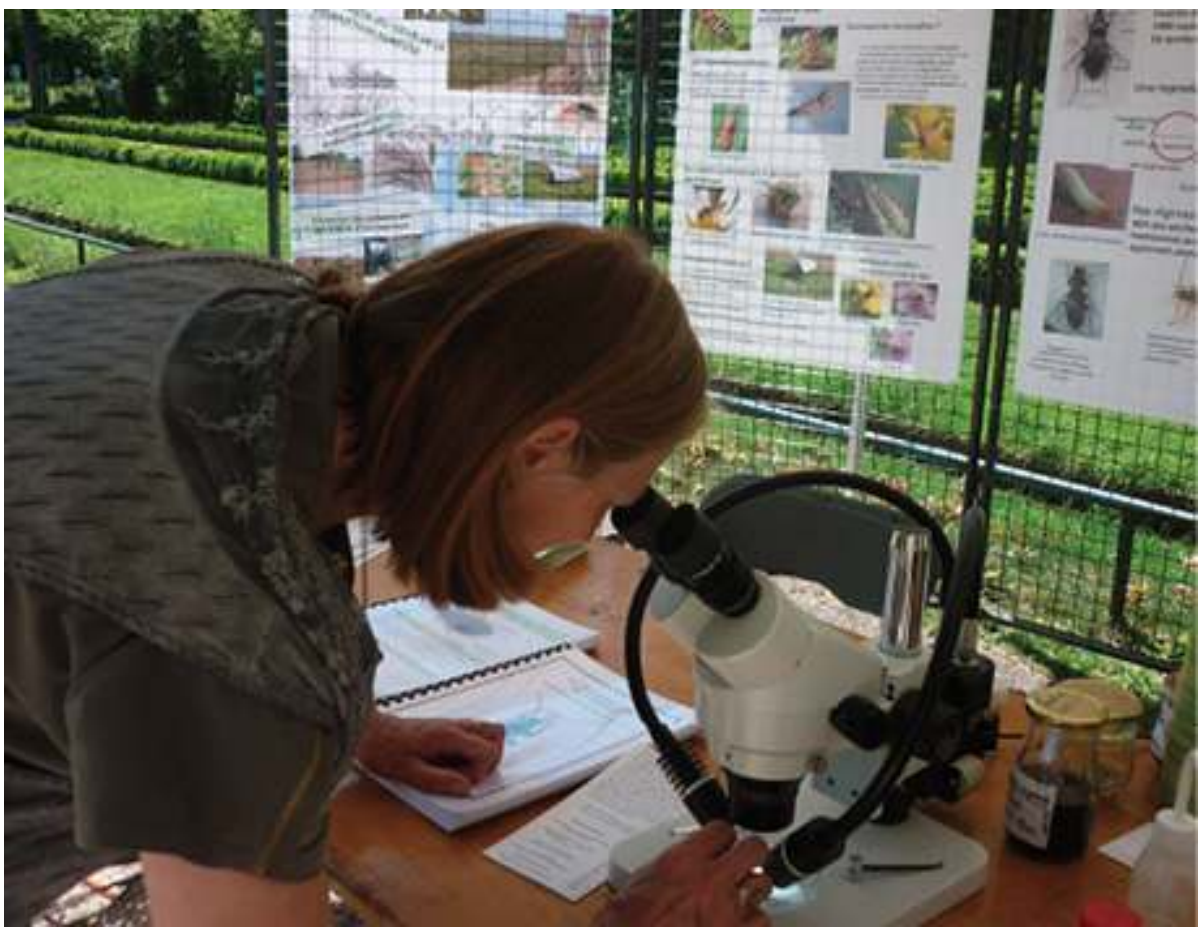

(c) Jardin des sciences de Dijon

30 Pourquoi vouloir absolument faire de ces institutions des vecteurs culturels alors que la médiation scientifique est au plus mal, que des notions scientifiques simples ne sont plus connues, que celles complexes comme les écosystèmes et les habitats ne sont ni appréhendées ni perçues par le public, ni du reste par beaucoup de naturalistes?

31 À plusieurs reprises lors de rencontres à l'occasion d'inspections, j'ai été frappé par le "fixisme " de certains naturalistes souhaitant toujours retrouver à la même place la même espèce, la même association. Sommes-nous aussi certains que les théories de Darwin soient bien comprises et assimilées ? Son $150^{\mathrm{e}}$ anniversaire, en 2009 , ne fut guère un déluge médiatique, du moins en France !

Nous sommes obnubilés par les espèces, et encore souvent par quelques espèces médiatiques, mais où se situe alors la dynamique des habitats? Qui aborde l'évolution de la phénologie des plantes et du climat? Qui se soucie de montrer à la fois le rôle bénéfique de bien des êtres exotiques et les limites de leur naturalisation? Qui doit faire passer ces discours pour rendre lisibles des concepts scientifiques et philosophiques complexes afin qu'ils soient appropriables par la société ? L'écologie traditionnelle avec son concept de climax, n'est plus d'actualité. La diversité biologique a introduit d'autres termes de référence. Qui se soucie d'apporter un peu de rigueur dans les discours et les publicités dans lesquels on confond allégrement diversité spécifique et diversité biologique, la défense des habitats et la coloration esthétique des bords de routes? Qui se préoccupe de la destruction progressive au nom du Développement durable, de la diversité biologique des prairies et accotements routiers en faisant non du fauchage, avec exportation de matière mais du gyrobroyage avec un enrichissement progressif en matière organique? Les sujets sont nombreux, très divers et peuvent être abordés de multiples manières. 

mutualisent leurs compétences et souhaitent émettre des discours communs, cohérents sur la science, la défense et la protection de la nature.

\section{Jardins botaniques et muséums doivent rapidement penser leur avenir}

Avant de se poser la question du rattachement administratif des jardins botaniques et muséums dans l'organisation de la collectivité qui en a la charge, de nombreuses autres questions doivent être posées. Muséums et jardins botaniques sont-ils les vecteurs d'une science obsolète, ou les porteurs d'une science en devenir dans le cadre des révolutions conceptuelles actuelles? Doivent-ils être des lieux de sciences et de transmission scientifique, ou des lieux à orientation artistique et d'apprentissage à l'esthétique? Les collections taxonomiques sont-elles des collections de références, pédagogiques ou constituent-elles un simple magasin d'objets pour exposition?

Il ne faudrait pas oublier que jardins botaniques et muséums sont porteurs d'un double message: celui de l'histoire des sciences naturelles et de la connaissance des objets animés et inanimés (géologie, paléontologie) avec des collections, des herbiers, des bibliothèques, des photothèques... mais aussi et surtout, celui d'une science en devenir, celle issue des nouveaux paradigmes. Or, ces nouvelles approches scientifiques sont-elles vraiment bien assimilées et en a-t-on tiré toutes les conséquences quant à la valeur des collections des diverses institutions? A-t-on bien perçu les conséquences de cette remise en cause d'une partie de l'écologie traditionnelle dans laquelle il était enseigné que toutes les associations tendaient vers leur point d'équilibre, le climax, alors que la diversité biologique c'est-à-dire ce complexe associant génome, espèces et habitats, insiste sur cette vision non statique de la nature avec sa dynamique, ses modifications, son adaptation, son évolution?

En effet, nous vivons dans un paradoxe, celui d'une information immédiatement disponible mais sans appareil critique à cause d'une connaissance insuffisante voire une méconnaissance de bien des fonctionnements de ce monde vivant auquel nous appartenons.

C'est sans doute l'un des défis que doivent rapidement relever les jardins botaniques et muséums, participer à la connaissance scientifique, participer à la diffusion de la philosophie des sciences et à l'éveil aux sciences, mais surtout transmettre la relativité de la stabilité des associations vivantes, animales comme végétales, dans l'espace comme dans le temps y compris avec les doutes, les interrogations, voire les manies et les idées reçues du monde scientifique.

Nous changeons de référence scientifique, que ces institutions nées sous d'autres siècles sachent sortir du poids de l'histoire pour prendre à bras le corps l'avenir. C'est un beau défi et il peut, mais surtout doit être relevé, les expériences de plusieurs institutions ici et là prouvent que cela est possible. 


\section{NOTES}

1. Allain, Y.-M., Une histoire des jardins botaniques, entre science et art paysager. Versailles : Éditions Quæ, 2012, 112 p.

2. Voir à ce sujet les deux études publiées par l'Observatoire du Patrimoine et de la Culture Scientifique et Technique de l'OCIM : Plateforme OCIM-Muséums, données 2010. Dijon : OCIM, 2012, 118 p. Enquête "Les jardins botaniques", données 2010. Dijon : OCIM, 2012, 136 p.

3. Allain, Y.-M., Les plantes exotiques, une réputation perdue ? Saint-Nazaire : Éditions Petit Génie, 2014, $138 \mathrm{p}$.

4. Notre avenir à tous, rapport Brundtland, publié par la commission des Nations Unies sur l'environnement et le développement, en 1987 et disponible en ligne sur www.mediaterre.org.

5. Bonneuil, C. et Fressoz, J.-B. L'évènement anthropocène. La Terre, l'histoire et nous. Paris : Le Seuil, 2013, $320 \mathrm{p}$.

6. Wilson, E.-O. The Biological Diversity crisis, Bioscience, $\mathrm{n}^{\circ} 11,1985$, pp. 700-706.

7. Bœuf, G., Allain, Y.-M. et Bouvier, M. L'apport des sciences participatives dans la connaissance de la biodiversité. Rapport remis à la Ministre en charge de l'Écologie, octobre 2011.

\section{RÉSUMÉS}

Après un bref rappel historique et une analyse de la situation des dernières décennies, l'auteur montre qu'aujourd'hui, l'un des principaux défis à relever par les muséums et jardins botaniques est de participer en synergie à la connaissance scientifique et à sa diffusion tout en transmettant les incertitudes et les interrogations actuelles sur le devenir des espèces végétales et animales.

\section{INDEX}

Mots-clés : développement durable, muséum, jardin botanique

\section{AUTEUR}

\section{YVES-MARIE ALLAIN}

ancien directeur du Jardin des plantes de Paris (Muséum national d'Histoire naturelle) et inspecteur général de l'environnement

ymallain@orange.fr 